\title{
Maternal dietary pattern characterised by high protein and low carbohydrate intake in pregnancy is associated with a higher risk of gestational diabetes mellitus in Chinese women: a prospective cohort study
}

\author{
Xuezhen Zhou ${ }^{1}$, Renjuan Chen ${ }^{1}$, Chunrong Zhong ${ }^{1}$, Jiangyue $\mathrm{Wu}^{1}$, Xiating $\mathrm{Li}^{1}$, Qian $\mathrm{Li}^{1}$, Wenli Cui ${ }^{1}$, \\ Nianhua $\mathrm{Yi}^{2}$, Mei Xiao ${ }^{2}$, Heng Yin ${ }^{2}$, Guoping Xiong ${ }^{3}$, Weizhen $\mathrm{Han}^{3}$, Liping Hao ${ }^{1}$, Xuefeng Yang ${ }^{1}$, \\ and Nianhong Yang ${ }^{1 *}$ \\ ${ }^{1}$ Hubei Key Laboratory of Food Nutrition and Safety, MOE Key Laboratory of Environment and Health, Department of \\ Nutrition and Food Hygiene, School of Public Health, Tongji Medical College, Huazhong University of Science and \\ Technology, 13 Hangkong Road, Wuhan, Hubei 430030, People's Republic of China \\ ${ }^{2}$ Department of Obstetrics and Gynecology, Hubei Maternal and Child Health Hospital, Wuhan, Hubei 430070, People's \\ Republic of China \\ ${ }^{3}$ Department of Obstetrics and Gynecology, The Central Hospital of Wuban, Hubei 430014, People's Republic of China
}

(Submitted 2 January 2018 - Final revision received 8 April 2018 - Accepted 6 August 2018)

\section{Abstract}

Maternal dietary patterns and macronutrients intake have been shown to affect the development of gestational diabetes mellitus (GDM), but the findings are inconsistent. We aimed to identify maternal dietary patterns and examine their associations with GDM risk, and to evaluate the contributions of macronutrients intake to these associations. We included 2755 Chinese pregnant women from the Tongji Maternal and Child Health Cohort. Dietary intakes were assessed using a validated semi-quantitative FFQ 2 weeks before the diagnosis of GDM. GDM ( $n$ 248) was diagnosed based on the results of a 75-g, 2-h oral glucose tolerance test at 24-28 weeks gestation. We derived five different dietary patterns from a principal component analysis. The results showed that high fish-meat-eggs scores, which were positively related to protein intake and inversely related to carbohydrate intake, were associated with a higher risk of GDM (adjusted OR for quartile $4 v$. quartile 1: 1.83; $95 \%$ CI 1.21, $\left.2 \cdot 79 ; P_{\text {trend }}=0.007\right)$ and higher plasma glucose levels. In contrast, high rice-wheat-fruits scores, which were positively related to carbohydrate intake and inversely related to protein intake, were associated with lower risk of GDM (adjusted OR for quartile $3 v$. quartile $1: 0 \cdot 54 ; 95 \%$ CI $\left.0 \cdot 36,0 \cdot 83 ; P_{\text {trend }}=0 \cdot 010\right)$ and lower plasma glucose levels. In addition, dietary protein and carbohydrate intake significantly contributed to the associations between dietary patterns and GDM risk or glucose levels. These findings suggest that a dietary pattern characterised by high protein and low carbohydrate intake in pregnancy was associated with a higher risk of GDM, which may provide important clues for dietary guidance during pregnancy to prevent GDM.

Key words: Dietary patterns: Gestational diabetes mellitus: Plasma glucose: Macronutrients

Gestational diabetes mellitus (GDM), defined as glucose intolerance with onset or first recognition during pregnancy, is a common pregnancy complication. GDM reflects a continuum risk of short-term and long-term adverse health outcomes for both mothers and their offspring ${ }^{(1-4)}$. During past decades, its prevalence has increased substantially across a range of multiethnic populations. In China, approximately $10 \%$ of all pregnancies have been reported to be complicated by $\mathrm{GDM}^{(5-7)}$.

Maternal diet plays an important role in the development of $\mathrm{GDM}^{(8-14)}$. In addition to individual nutrients and specific food groups, such as cholesterol, saturated and trans fats, haem Fe, red and processed meats, several dietary patterns have been identified to be linked to GDM risk ${ }^{(15-21)}$. Only a few studies have examined the association between dietary patterns during pregnancy and risk of $\mathrm{GDM}^{(17-21)}$. Results from these studies provided an evidence base for dietary recommendation to prevent GDM, but the findings were compromised by either relatively small sample size ${ }^{(17,18)}$ or variance in study design ${ }^{(19)}$, dietary assessment tools ${ }^{(20,21)}$ and diagnostic criteria for $\mathrm{GDM}^{(20,21)}$.

Composition and quality of macronutrients may contribute to the association between dietary patterns and development of GDM. Dietary patterns associated with reduced risk of GDM are

Abbreviations: GDM, gestational diabetes mellitus; NHSII, Nurses' Health Study II; OGTT, oral glucose tolerance test; TMCHC, Tongji Maternal and Child Health Cohort.

* Corresponding authors: X. Yang, email xxyxf@mails.tjmu.edu.cn; N. Yang, email zynh@mails.tjmu.edu.cn 
often characterised by high consumption of carbohydrates, mainly complex ones, and low consumption of animal protein and animal fat ${ }^{(18,19,21)}$. However, there are only a limited number of studies on this issue and the results are inconclusive. Take protein for example, results from a multiethnic Asian cohort suggested that higher intakes of both animal and vegetable protein during pregnancy were associated with a higher risk of $\mathrm{GDM}^{(22)}$, in contrast with the findings from the cohort of US nurses, which showed that among women of reproductive age, substitution of vegetable protein for animal protein may potentially lower GDM risk ${ }^{(23)}$.

Therefore, the aim of this study was to identify maternal dietary patterns and examine their associations with GDM using data from a large prospective cohort of pregnant women in central China, furthermore, to evaluate the contributions of macronutrients intake to the associations between dietary patterns and GDM risk.

\section{Methods \\ Study design}

The Tongji Maternal and Child Health Cohort (TMCHC) study is an ongoing population-based prospective cohort to investigate the association between maternal diet and the health outcomes of mother and offspring ${ }^{(24)}$. The TMCHC study was established in January 2013 and enrolled healthy pregnant women at 8-16 weeks of gestation when they attended their first antenatal visit at a maternity clinic in one of three public hospitals in Wuhan, China. We included TMCHC participants in this analysis if they reported a singleton pregnancy, completed an oral glucose tolerance test (OGTT), and completed the FFQ before their OGTT. Women who developed GDM in a previous pregnancy or had a previous diagnosis of type 2 diabetes or whose fasting plasma glucose value in this early pregnancy $\geq 5 \cdot 1 \mathrm{mmol} / 1$ were excluded because they may have changed their diets and lifestyles to prevent a recurrence of $\mathrm{GDM}^{(13)}$. Finally, a total of 2755 women were included in the analysis from September 2013 to May 2016 (online Supplementary Fig. S1). This study was approved by the ethics review committee of Tongji Medical College, Huazhong University of Science and Technology. All participants gave informed written consent upon recruitment. This study was registered at clinicaltrials.gov as NCT03099837.

\section{Dietary assessment}

The participants reported their dietary intakes during the past 4 weeks using a semi-quantitative FFQ, and completed FFQ 2 weeks before the diagnosis of GDM. The FFQ was validated in a subsample of TMCHC cohort participants, showing that it is a reasonably reliable and valid tool for assessing most food and nutrient intakes of pregnant women in urban areas of central China $^{(24)}$. The FFQ consisted of sixty-one food items based on the food nutrient composition and eating habits of Chinese individuals, covering more than 200 kinds of food. For each item, trained interviewers conducted face-to-face interviews, the participants were asked to recall the frequency of intake and portion size consumed during the past 4 weeks. To reduce the influence of food intakes with large variances at the expense of those with minor variances ${ }^{(25)}$, we standardised the sixty-one food items and assembled them into sixteen non-overlapping food groups: rice and wheat products, whole grains, poultry, red meat, animal organs and blood, freshwater fish, seafood, eggs, beans and bean products, leafy and cruciferous vegetables, root vegetables, melons and solanaceous vegetables, mushrooms and algae, fruits, nuts, and dairy products. Daily intakes of nutrient and energy were calculated based on the continuously updated China Food Composition Database ${ }^{(26)}$, and each energy-yielding macronutrient intake was expressed as the percentage of total energy intake using the nutrient-density $\operatorname{method}^{(14,27)}$.

\section{Ascertainment of gestational diabetes mellitus}

The primary outcome was GDM. At 24-28 weeks, participants were routinely offered $75 \mathrm{~g}, 2$-h OGTT. All participants had overnight fasting of at least $8 \mathrm{~h}$ before OGTT. Fasting plasma glucose and 1- and 2-h post-load plasma glucose levels were measured by enzymatic assays using an automated biochemical analyser. GDM was diagnosed when any of the glucose values during the diagnostic OGTT met or exceeded the criteria as recommended by the International Association of Diabetes and Pregnancy Study Group: fasting plasma glucose $\geq 5.1 \mathrm{mmol} / \mathrm{l}$ or 1 -h plasma glucose $\geq 10.0 \mathrm{mmol} / \mathrm{l}$ or 2 -h plasma glucose $\geq 8.5 \mathrm{mmol} / \mathrm{l}^{(28)}$.

\section{Covariates}

Covariates were assessed using a structured questionnaire at the enrollment interview. Maternal age (in years) was treated as a continuous variable, except for the descriptive statistics for which we divided age into four groups ( $\leq 24,25-29,30-35$ and 236). Ethnicity was divided into two categories (Han Chinese, others). Educational attainment was recorded as the number of completed years of schooling and divided into four categories $(\leq 9,10-12,13-15$ and $\geq 16$ ). Average personal income (per month, Chinese Yuan) was divided into five categories $(\leq 1000$, 1001-2999, 3000-4999, 5000-9999 and $\geq 10000$ ). Pre-pregnancy BMI $\left(\mathrm{kg} / \mathrm{m}^{2}\right)$, which was calculated from self-reported prepregnancy weight ${ }^{(29)}$ and measured height, was divided into four categories $(<18.5, \quad 18.5-23.9,24.0-27.9$ and $\geq 28.0$ ) according to the Chinese BMI cutoffs ${ }^{(30)}$. Gestational weight gains before GDM diagnosis were calculated by subtracting selfreported pre-pregnancy weights from weights measured at OGTT test. Parity were each divided into two categories $(0, \geq 1)$. The other covariates, including smoking habits, alcohol consumption habits, family history of diabetes and family history of obesity, were treated as dichotomised variables (yes/no).

\section{Statistical analysis}

Principal component analysis with varimax rotation was used to derive the dietary patterns ${ }^{(15,31)}$. Sensitivity analyses were performed to review the reproducibility and stability of the pattern extraction. Principal component analysis is a data-driven technique that reduces the dimensions of the data and groups correlated variables to identify common factors (i.e. dietary patterns) underlying food consumption ${ }^{(25,31,32)}$. A total of five 
factors were determined in this study, and the number of factors retained was based on the eigenvalue, factor interpretability after varimax rotation and a scree plot showing the proportion of the variance of total consumption of the food variables ${ }^{(15,25,33)}$. The dietary pattern score for each participant was calculated by summing the standardised frequency of food groups weighted by their factor loadings. Each woman received a factor score for each identified pattern, the mean factor score for each pattern is zero, and higher dietary pattern scores indicate greater adherence to the derived pattern ${ }^{(15)}$. We used dietary pattern scores to rank participants according to the degree to which they conformed to each dietary pattern, and divided dietary pattern scores into quartiles for further analysis. In this study, the food items with loadings of 0.40 or higher on a factor were considered important to the interpretability of each pattern.

The OR and 95\% CI of GDM for each category of dietary pattern compared with the lowest category were estimated using logistic regression models. In addition, the OR and $95 \%$ CI of GDM associated with per SD increment in the dietary pattern scores were also calculated. Stratified analyses were conducted by BMI $(<18 \cdot 5 / 18 \cdot 5-23 \cdot 9 / \geq 24 \cdot 0)$ and family history of diabetes (yes/no), and other covariates mentioned above were also considered in additional stratified analyses (data not shown). Multivariate linear regression models were estimated to assess the association between dietary patterns and plasma glucose levels. To explore potential dietary contributors for the association between dietary patterns and GDM, we adjusted for macronutrients (e.g. total fat, animal fat, vegetable fat, total protein, animal protein, vegetable protein, total carbohydrate and ratio of total protein to total carbohydrate), other nutrients (e.g. dietary fibre, cholesterol, vitamin C, vitamin E, Mg, K, Ca, total Fe, haem Fe and non-haem $\mathrm{Fe}$ ), and each food or food group component of each dietary pattern.
Comparisons between groups were performed using $\chi^{2}$ tests for categorical variables, ANOVA or $t$ tests for continuous variables with normal distribution, or non-parametric MannWhitney and Kruskal-Wallis tests for continuous variables with skewed distributions. Frequencies and percentages were used to describe the distributions of categorical variables, and the means and standard deviations were used to describe the distribution of continuous variables. To quantify a linear trend, we assigned the median value for each category and treat the median values as continuous variable in the logistic regression model. All tests of statistical significance were two-sided, and $P<0.05$ was considered statistically significant. All statistical analyses were performed using SPSS version 21.0 (IBM Corporation). All data collected were double entered into the EpiData software program ${ }^{(24)}$

\section{Results}

\section{Dietary patterns}

In all, five distinct dietary patterns with eigenvalues above $1 \cdot 0$, as well as factor loadings above 0.40 , were extracted from the scree plot (Table 1, online Supplementary Fig. S2). These five patterns accounted for $45.6 \%$ of the total variation in food frequency intakes. The first pattern, beans-vegetables, included higher intakes of root vegetables, melons and solanaceous vegetables, mushrooms and algae, beans and bean products (soyabean, mung bean, soyabean milk), and leafy and cruciferous vegetables. The second pattern, nuts-whole grains, had higher intakes of nuts, whole grains and dairy products (milk, milk powder and yogurt). The third pattern, organs-poultryseafood, had higher intakes of animal organs, blood, seafood and poultry. The fourth pattern, fish-meat-eggs, had higher

Table 1. Factor loading matrix for the five major dietary patterns identified by principal component analysis using varimax rotation in Tongji Maternal and Child Health Cohort study $(n$ 2755)*

\begin{tabular}{|c|c|c|c|c|c|}
\hline \multirow[b]{2}{*}{ Food groups } & \multicolumn{5}{|c|}{ Dietary patterns } \\
\hline & Beans-vegetables & Nuts-whole grains & Organs-poultry-seafood & Fish-meat-eggs & Rice-wheat-fruits \\
\hline Root vegetables & 0.71 & - & - & - & - \\
\hline Melon and solanaceous vegetables & 0.60 & - & - & - & - \\
\hline Mushrooms and algae & 0.59 & - & - & - & - \\
\hline Beans and bean products $\dagger$ & 0.54 & - & - & - & - \\
\hline Leafy and cruciferous vegetables & 0.42 & - & - & - & - \\
\hline Nuts & - & 0.70 & - & - & - \\
\hline Whole grains & - & 0.55 & - & - & - \\
\hline Dairy products $\ddagger$ & - & 0.45 & - & - & - \\
\hline Animal organs and blood & - & - & 0.67 & - & - \\
\hline Seafood & - & - & 0.61 & - & - \\
\hline Poultry & - & - & 0.60 & - & - \\
\hline Freshwater fishes & - & - & - & 0.63 & - \\
\hline Red meat & - & - & - & 0.62 & - \\
\hline Eggs & - & - & - & 0.59 & - \\
\hline Rice and wheat products & - & - & - & - & 0.82 \\
\hline Fruits & - & - & - & - & 0.56 \\
\hline Cumulative variance explained $(\%) \S$ & $12 \cdot 0$ & $21 \cdot 0$ & 29.9 & 38.6 & $45 \cdot 6$ \\
\hline
\end{tabular}

* Values are factor loadings (correlation coefficients) between each food frequency variable and the dietary pattern derived from principal component analysis. Food groups are sorted by size of loading coefficients. Absolute values $<0.40$ were not listed for simplicity.

$\dagger$ Soyabean, mung bean, soyabean milk, bean curd, and so on.

‡ Milk, milk powder and yogurt.

$\S$ Percentage of variance in total food intake explained by patterns. 
intakes of freshwater fishes, red meat and eggs. The fifth pattern, rice-wheat-fruits, had higher intakes of rice, wheat products and fruits.

\section{Dietary patterns in relation to maternal characteristics}

The characteristics of the participants are presented in Table 2 . We observed that the beans-vegetables pattern score increased with education level and was higher in women with a family history of obesity, in non-smokers and in multiparous women, while the score was lower in the young ( $\leq 24$ years) and older ( $\geq 36$ years) age groups. The nuts-whole grains score increased with maternal age, education level and average personal income, while the score was lower in smokers, in women who habitually consumed alcohol, and in multiparous women. The organs-poultry-seafood pattern scores increased with education level and decreased with increasing pre-pregnancy BMI and were higher in women who consumed alcohol. The fishmeat-eggs pattern score was higher in the few ( $\leq 9$ years) and high ( $\geq 16$ years) education groups. The rice-wheat-fruits pattern score was lower in the older age groups, in women with a family history of diabetes, and decreased with increasing prepregnancy BMI.

We also analysed the characteristics of the 2755 women according to the quartile of the dietary pattern score and obtained similar results (online Supplementary Table S3).

\section{Dietary patterns in relation to gestational diabetes mellitus}

GDM occurred in 248 (9.0\%) of 2755 pregnant women. For the fish-meat-eggs pattern, the adjusted OR for the highest quartile compared with the lowest was 1.83 (95\% CI 1.21, 2.79; $\left.P_{\text {trend }}=0.007\right)$; each sD of fish-meat-eggs score was associated with a $32(95 \%$ CI 12, 54) \% increment in OR of GDM. For the rice-wheat-fruits dietary pattern, we found that the adjusted OR for the third quartile compared with the lowest was 0.54 (95\% CI 0.36, 0.83; $\left.P_{\text {trend }}=0 \cdot 010\right)$; each SD of rice-wheat-fruits score was associated with a $22(95 \%$ CI 12,32$) \%$ decrement in OR of GDM. No associations were seen for the beans-vegetables, nuts-whole grains or organs-poultry-seafood patterns in relation to GDM (Table 3).

We performed stratified analyses according to the prepregnancy BMI $(<18 \cdot 5 / 18 \cdot 5-23 \cdot 9 / \geq 24 \cdot 0)$ and the family history of diabetes (yes/no). We found that in the high BMI $(\geq 24 \cdot 0)$ group, a high fish-meat-eggs score was associated with a significantly increased risk of GDM for the highest quartile $v$. the lowest quartile $\left(2.84,95 \%\right.$ CI $\left.1.03,7.80 ; P_{\text {trend }}=0.021\right)$, and each SD of the fish-meat-eggs score was associated with a 62 (95\% CI 12, 134) \% increment in OR of GDM, while for ricewheat-fruits dietary pattern, no significant association was observed in the high BMI $(\geq 24 \cdot 0)$ group (online Supplementary Table S4). Similarly results were obtained in women with a family history of diabetes: the positive association between fish-meat-eggs pattern and the risk of GDM was stronger, while for rice-wheat-fruits dietary pattern, no significant association was observed in women with a family history of diabetes (online Supplementary Table S4).
Online Supplementary Table S5 shows participants' macronutrient intakes according to the quartiles of dietary pattern scores. The fish-meat-eggs pattern was positively associated with a high intake of energy content from total protein, animal protein, total fat, animal fat and a high ratio of total protein to total carbohydrate, while inversely associated with a high intake of energy content from carbohydrate, vegetable protein and vegetable fat. In addition, we also observed that the ricewheat-fruits score was positively related to the intake of energy content from carbohydrate, while being inversely related to intake of energy content from total protein, animal protein, vegetable protein, total fat, vegetable fat and ratio of total protein to total carbohydrate.

We further investigated the contributions of energy-yielding macronutrients on the association between dietary patterns and risk of GDM (Table 4). Total carbohydrate intake was inversely associated with risk of GDM, while total fat, animal fat, total protein, animal protein intake and ratio of total protein to total carbohydrate were positively associated with risk of GDM. The association of the fish-meat-eggs score with GDM risk for comparisons of highest with lowest quartiles was no longer significant after additional adjustment for total protein (percentage of energy) (OR 1.42; 95\% CI 0.90, 2.22), or animal protein (percentage of energy) (OR 1.51; $95 \%$ CI 0.94, 2.44), or total carbohydrate (percentage of energy) (OR 1.48; 95\% CI 0.92, 2.36), or ratio of total protein to total carbohydrate (OR 1.35; $95 \%$ CI 0.84, 2.16), which indicated that protein and carbohydrate may be the main contributors to the observed association between the fish-meat-eggs score and GDM risk. Moreover, for the rice-wheat-fruits dietary pattern, the association with GDM was attenuated substantially after adjusting for total protein intake and ratio of total protein to total carbohydrate, respectively.

In addition, we performed similar analyses for both dietary patterns score by adjusting for other nutrients (e.g. dietary fibre, cholesterol, vitamin $\mathrm{C}$, vitamin $\mathrm{E}, \mathrm{Mg}, \mathrm{K}$, Ca, total Fe, haem Fe and non-haem $\mathrm{Fe}$ ), and each food or food group component of each dietary pattern. However, these adjustments, except for cholesterol and fruits, did not substantially alter the association.

\section{Dietary patterns in relation to plasma glucose levels}

Multivariate linear regression models were constructed to assess the association of dietary patterns with plasma glucose levels in Table 5. The adjusted results showed that per SD increase in the fish-meat-eggs score, the fasting, 1-h and 2-h plasma glucose reading increased by $0.018 \mathrm{mmol} / 1$ (95\% CI $0.004,0.033)$, $0.136 \mathrm{mmol} / 1(95 \% \mathrm{CI} 0.077,0.195)$ and $0.074 \mathrm{mmol} / 1(95 \% \mathrm{CI}$ $0.028,0.120)$, respectively. With an sD increase in the ricewheat-fruits score, fasting, 1 -h and 2 -h plasma glucose decreased by $0.014 \mathrm{mmol} / 1 \quad(95 \%$ CI $-0.028,-0.001)$, $0 \cdot 100 \mathrm{mmol} / \mathrm{l}(95 \% \mathrm{CI}-0 \cdot 158,-0.042)$ and $0.093 \mathrm{mmol} / \mathrm{l}(95 \%$ CI $-0 \cdot 137,-0 \cdot 048)$, respectively.

An inverse association was found between plasma glucose levels and total carbohydrate intake, and a positive association was found between plasma glucose levels and total fat, animal fat, total protein, animal protein intake and ratio of total protein to total carbohydrate, respectively. For both dietary patterns, the association with fasting plasma glucose was attenuated 
Table 2. Dietary pattern scores, according to maternal characteristics, in 2755 participants in Tongji Maternal and Child Health Cohort study* (Numbers and percentages; mean values and standard deviations)

\begin{tabular}{|c|c|c|c|c|c|c|c|c|c|c|c|c|}
\hline \multirow[b]{3}{*}{ Characteristics } & \multirow{2}{*}{\multicolumn{2}{|c|}{ All participants }} & \multicolumn{10}{|c|}{ Dietary patterns } \\
\hline & & & \multicolumn{2}{|c|}{$\begin{array}{l}\text { Beans- } \\
\text { vegetables }\end{array}$} & \multicolumn{2}{|c|}{ Nuts-whole grains } & \multicolumn{2}{|c|}{$\begin{array}{l}\text { Organs-poultry- } \\
\text { seafood }\end{array}$} & \multicolumn{2}{|c|}{$\begin{array}{l}\text { Fish-meat- } \\
\text { eggs }\end{array}$} & \multicolumn{2}{|c|}{$\begin{array}{l}\text { Rice-wheat- } \\
\text { fruits }\end{array}$} \\
\hline & $n$ & $\%$ & Mean & SD & Mean & SD & Mean & SD & Mean & SD & Mean & SD \\
\hline \multicolumn{13}{|c|}{ Age at enrollment (years) } \\
\hline $17-24$ & 311 & 11.3 & -0.17 & 0.99 & $-0 \cdot 16$ & 0.93 & -0.19 & 0.97 & -0.01 & $1 \cdot 11$ & 0.14 & 1.05 \\
\hline $25-29$ & 1641 & 59.6 & 0.00 & 0.99 & 0.00 & 0.98 & 0.02 & 0.99 & -0.03 & 0.99 & 0.02 & 0.98 \\
\hline $30-35$ & 691 & $25 \cdot 1$ & 0.09 & 0.99 & 0.04 & 1.05 & 0.05 & 1.03 & 0.06 & 0.95 & -0.11 & 1.05 \\
\hline $36-45$ & 112 & 4.0 & -0.12 & $1 \cdot 12$ & 0.26 & 1.07 & -0.05 & 0.95 & 0.12 & 1.04 & -0.07 & 0.73 \\
\hline$P$ & \multicolumn{2}{|c|}{-} & \multicolumn{2}{|c|}{0.001} & \multicolumn{2}{|c|}{$<0.001$} & \multicolumn{2}{|c|}{0.004} & \multicolumn{2}{|c|}{0.146} & \multicolumn{2}{|c|}{0.001} \\
\hline Ethnicity & & & & & & & & & & & & \\
\hline Han Chinese & 2682 & 97.4 & 0.00 & 1.00 & 0.00 & 1.00 & 0.00 & 1.00 & 0.00 & 1.00 & 0.00 & 1.01 \\
\hline Others & 73 & 2.6 & 0.18 & 1.00 & 0.00 & 0.90 & -0.16 & 1.02 & 0.12 & 0.96 & 0.06 & 0.62 \\
\hline$P$ & - & & 0.1 & & 0.5 & & & & 0.3 & & & \\
\hline Education level & & & & & & & & & & & & \\
\hline$\leq 9$ & 79 & $2 \cdot 9$ & -0.39 & 1.05 & -0.47 & 1.04 & -0.39 & 0.81 & 0.05 & 1.13 & 0.03 & 1.03 \\
\hline $10-12$ & 317 & 11.5 & -0.18 & 0.98 & -0.30 & 1.11 & -0.25 & 0.97 & 0.01 & $1 \cdot 10$ & -0.07 & 1.43 \\
\hline $13-15$ & 710 & $25 \cdot 7$ & -0.06 & 1.05 & -0.12 & 1.00 & 0.00 & 1.00 & -0.11 & 1.04 & 0.08 & 0.80 \\
\hline$\geq 16$ & 1589 & $57 \cdot 7$ & 0.09 & 0.96 & 0.13 & 0.95 & 0.07 & 1.01 & 0.05 & 0.95 & -0.03 & 0.98 \\
\hline Missing & 60 & $2 \cdot 2$ & -0.32 & 1.00 & 0.19 & 0.87 & -0.09 & 0.90 & -0.14 & 0.97 & $0 \cdot 10$ & 0.87 \\
\hline$P$ & - & & $<0$ & & $<0$ & & & & 0.0 & & $0 . c$ & \\
\hline Average person & & & & & & & & & & & & \\
\hline$\leq 1000$ & 10 & 0.4 & 0.24 & 0.83 & $-0 \cdot 27$ & 1.00 & 0.02 & $1 \cdot 11$ & -0.58 & 1.06 & 0.23 & 0.41 \\
\hline $1001-2999$ & 174 & $6 \cdot 3$ & -0.09 & 0.97 & -0.20 & 1.06 & -0.05 & 0.96 & -0.11 & $1 \cdot 11$ & -0.09 & $1 \cdot 21$ \\
\hline $3000-4999$ & 853 & 31.0 & -0.12 & 1.03 & -0.06 & 1.00 & -0.03 & 1.01 & -0.01 & 0.98 & 0.01 & 1.09 \\
\hline 5000-9999 & 1155 & 41.9 & 0.05 & 0.99 & 0.05 & 0.98 & -0.02 & 0.99 & -0.01 & 1.00 & -0.01 & 0.97 \\
\hline$\geq 10000$ & 512 & 18.6 & 0.15 & 0.94 & 0.06 & 1.00 & 0.12 & 1.00 & 0.08 & 0.96 & 0.04 & 0.67 \\
\hline Missing & 51 & 1.8 & -0.41 & 1.07 & 0.00 & 1.04 & -0.09 & 1.15 & 0.04 & 1.08 & -0.12 & 1.80 \\
\hline$P$ & - & & $<0$ & & 0.0 & & & & 0.1 & & & \\
\hline Family history of & & & & & & & & & & & & \\
\hline Yes & 236 & 8.6 & -0.04 & 0.98 & 0.11 & 0.98 & -0.05 & 1.04 & 0.08 & 1.07 & -0.24 & $1 \cdot 76$ \\
\hline No & 2467 & 89.5 & 0.01 & 1.00 & -0.01 & 1.00 & 0.01 & 1.00 & 0.00 & 0.99 & 0.02 & 0.90 \\
\hline Missing & 52 & 1.9 & -0.20 & 1.25 & -0.05 & 0.86 & -0.06 & 0.89 & -0.24 & 1.05 & 0.09 & 0.65 \\
\hline$P$ & - & & 0.2 & & 0.2 & & & & 0.1 & & & \\
\hline Family history of & & & & & & & & & & & & \\
\hline Yes & 47 & $1 \cdot 7$ & 0.19 & 0.95 & $-0 \cdot 18$ & $1 \cdot 12$ & 0.03 & 0.96 & -0.02 & 1.09 & $-0 \cdot 11$ & 0.89 \\
\hline No & 2641 & 95.9 & 0.01 & 1.00 & 0.00 & 1.00 & 0.00 & 1.00 & 0.00 & 1.00 & 0.00 & 1.01 \\
\hline Missing & 67 & 2.4 & -0.37 & 1.15 & -0.01 & 0.92 & -0.21 & 0.91 & -0.03 & 1.08 & 0.03 & 0.83 \\
\hline$P$ & - & & 0.0 & & 0.4 & & & & 0.9 & & & \\
\hline Smoking & & & & & & & & & & & & \\
\hline Yes & 449 & $16 \cdot 3$ & -0.11 & 1.02 & -0.14 & 1.03 & 0.01 & 1.05 & -0.04 & 1.05 & 0.00 & 0.90 \\
\hline No & 2306 & 83.7 & 0.02 & 0.99 & 0.03 & 0.99 & 0.00 & 0.99 & 0.01 & 0.99 & 0.00 & 1.02 \\
\hline$P$ & - & & 0.0 & & $0 . c$ & & & & 0.3 & & & \\
\hline Alcohol & & & & & & & & & & & & \\
\hline Yes & 409 & $14 \cdot 8$ & 0.02 & 0.94 & -0.11 & 0.94 & 0.10 & 0.97 & -0.06 & 1.02 & -0.04 & 1.07 \\
\hline No & 2346 & 85.2 & 0.00 & 1.01 & 0.02 & 1.01 & -0.02 & 1.00 & 0.01 & 1.00 & 0.01 & 0.99 \\
\hline$P$ & - & & 0.5 & & 0.0 & & & & 0.1 & & & \\
\hline Parity & & & & & & & & & & & & \\
\hline 0 & 2331 & $84 \cdot 6$ & -0.02 & 1.00 & 0.04 & 0.98 & -0.01 & 1.00 & -0.02 & 1.00 & 0.00 & 1.04 \\
\hline$\geq 1$ & 424 & $15 \cdot 4$ & 0.12 & 0.99 & -0.20 & 1.07 & 0.04 & 1.01 & 0.09 & 0.99 & -0.02 & 0.77 \\
\hline $\bar{P}$ & - & & 0.0 & & $<0$ & & & & 0.0 & & & \\
\hline Pre-pregnancy & & & & & & & & & & & & \\
\hline$<18.5$ & 523 & $19 \cdot 0$ & -0.06 & 0.98 & 0.03 & 0.97 & 0.06 & 1.01 & -0.01 & 1.02 & 0.16 & 0.65 \\
\hline $18 \cdot 5-23.9$ & 1920 & 69.7 & 0.02 & 0.99 & -0.01 & 0.99 & 0.01 & 1.00 & 0.02 & 0.97 & -0.02 & 1.07 \\
\hline $24 \cdot 0-27 \cdot 9$ & 271 & $9 \cdot 8$ & 0.00 & 1.03 & 0.02 & 1.09 & -0.16 & 0.97 & -0.10 & 1.09 & $-0 \cdot 10$ & 1.03 \\
\hline$\geq 28.0$ & 41 & 1.5 & -0.05 & 1.24 & -0.13 & 1.03 & -0.20 & 0.84 & 0.03 & 1.31 & -0.25 & 0.72 \\
\hline $\bar{P}$ & & & 0.4 & & 0.7 & & & & 0.3 & & & \\
\hline
\end{tabular}

CNY, Chinese Yuan.

* Dietary pattern scores created by multiplying factor loadings by corresponding standardised value for frequency intake of each food and adding all these items. Values are mean factor scores, derived by extraction of five dietary factors. Not adjusted for confounders.

$+1 \mathrm{CNY}=0.16$ US dollars.

$\ddagger$ Value 0 means never given birth before.

substantially and was not statistically significant after adjusting for total protein, animal protein, total carbohydrate intake and ratio of total protein to total carbohydrate. No large or statistically significant changes of $\beta$ (95\%) with 1- and 2-h plasma glucose after adjusting for any macronutrient intake for both dietary patterns were observed. 
Table 3. Dietary pattern scores associated with gestational diabetes mellitus (GDM) $(n 2755)^{*} \dagger$

(Odds ratios and $95 \%$ confidence intervals; numbers and percentages; mean values, minimum and maximum values)

\begin{tabular}{|c|c|c|c|c|c|c|c|c|c|c|c|c|}
\hline \multirow[b]{3}{*}{ Dietary patterns } & \multicolumn{8}{|c|}{ Quartiles of dietary pattern scores } & \multirow[b]{3}{*}{$P_{\text {for trend }}$} & & & \multirow[b]{3}{*}{$P$} \\
\hline & \multicolumn{2}{|c|}{ Q1 } & \multicolumn{2}{|r|}{ Q2 } & \multicolumn{2}{|r|}{ Q3 } & \multicolumn{2}{|r|}{ Q4 } & & \multicolumn{2}{|c|}{ Per sD increase } & \\
\hline & OR & $95 \% \mathrm{Cl}$ & OR & $95 \% \mathrm{Cl}$ & OR & $95 \% \mathrm{Cl}$ & OR & $95 \% \mathrm{Cl}$ & & OR & $95 \% \mathrm{Cl}$ & \\
\hline \multicolumn{13}{|l|}{ Beans-vegetables } \\
\hline \multicolumn{13}{|l|}{ GDM } \\
\hline$n$ & & \multicolumn{2}{|r|}{46} & \multicolumn{2}{|r|}{71} & \multicolumn{2}{|r|}{60} & & & & \\
\hline$\%$ & \multirow{2}{*}{\multicolumn{2}{|c|}{$10 \cdot 3$}} & \multicolumn{2}{|r|}{$6 \cdot 7$} & \multirow{2}{*}{\multicolumn{2}{|c|}{$10 \cdot 3$}} & \multicolumn{2}{|r|}{$8 \cdot 7$} & & & & \\
\hline Mean & & & & -0.30 & & 0.37 & & 1.23 & & & & \\
\hline Min, max & & -0.66 & & $66,0.04$ & & $4,0.70$ & & $70,3 \cdot 72$ & & & & \\
\hline Model 1 & 1 & Ref. & 0.62 & $0.42,0.92$ & 1.00 & $0.71,1.41$ & 0.83 & $0.58,1.19$ & 0.731 & 0.98 & $0.86,1 \cdot 12$ & 0.747 \\
\hline Model 2 & 1 & Ref. & 0.63 & $0.43,0.94$ & 1.03 & $0.73,1.47$ & 0.84 & $0.58,1.21$ & 0.847 & 1.00 & $0.87,1.14$ & 0.940 \\
\hline Model 3 & 1 & Ref. & 0.68 & $0.44,1.04$ & $1 \cdot 15$ & $0.78,1.68$ & 0.97 & $0.64,1.46$ & 0.649 & 1.04 & $0.90,1 \cdot 21$ & 0.592 \\
\hline Nuts-whole grain & & & & & & & & & & & & \\
\hline GDM & & & & & & & & & & & & \\
\hline$n$ & & 68 & & 51 & & 55 & & 74 & & & & \\
\hline$\%$ & & 9 & & 7.4 & & 8.0 & & $10 \cdot 7$ & & & & \\
\hline Mean & & 1.33 & & -0.28 & & 0.40 & & 1.20 & & & & \\
\hline Min, $\max$ & & -0.65 & & $65,0.09$ & & $9,0 \cdot 72$ & & $72,3.06$ & & & & \\
\hline Model 1 & 1 & Ref. & 0.73 & $0.50,1.07$ & 0.79 & $0.55,1.15$ & $1 \cdot 10$ & $0.78,1.55$ & 0.595 & 1.04 & $0.91,1.18$ & 0.593 \\
\hline Model 2 & 1 & Ref. & 0.77 & $0.53,1.13$ & 0.84 & $0.58,1.22$ & 1.14 & $0.80,1.61$ & 0.419 & 1.06 & $0.93,1.21$ & 0.353 \\
\hline Model 3 & 1 & Ref. & 0.83 & $0.55,1.26$ & 0.89 & $0.59,1.35$ & 1.25 & $0.84,1.86$ & 0.308 & 1.09 & $0.94,1.26$ & 0.245 \\
\hline $\begin{array}{l}\text { Organs-poultry-s } \\
\text { GDM }\end{array}$ & & & & & & & & & & & & \\
\hline$n$ & & 72 & & 61 & & 54 & & 61 & & & & \\
\hline$\%$ & & 0.5 & & 8.9 & & 7.8 & & 8.9 & & & & \\
\hline Mean & & 1.24 & & -0.38 & & 0.29 & & 1.32 & & & & \\
\hline Min, $\max$ & & -0.73 & -0 . & $73,-0.05$ & & $05,0.66$ & & $6,3 \cdot 68$ & & & & \\
\hline Model 1 & 1 & Ref. & 0.83 & $0.58,1.19$ & 0.73 & $0.50,1.05$ & 0.83 & $0.58,1.19$ & 0.260 & 0.94 & $0.82,1.07$ & 0.329 \\
\hline Model 2 & 1 & Ref. & 0.84 & $0.59,1.21$ & 0.73 & $0.50,1.05$ & 0.86 & $0.60,1.23$ & 0.328 & 0.95 & $0.83,1.08$ & 0.430 \\
\hline Model 3 & 1 & Ref. & 0.93 & $0.63,1.39$ & 0.84 & $0.57,1.26$ & 1.01 & $0.68,1.51$ & 0.918 & 1.01 & $0.87,1.17$ & 0.900 \\
\hline Fish-meat-eggs & & & & & & & & & & & & \\
\hline GDM & & & & & & & & & & & & \\
\hline$n$ & & 53 & & 56 & & 60 & & 79 & & & & \\
\hline$\%$ & & .7 & & $8 \cdot 1$ & & $8 \cdot 7$ & & 11.5 & & & & \\
\hline Mean & & 1.31 & & -0.24 & & 0.36 & & 1.19 & & & & \\
\hline Min, $\max$ & &,-0.60 & & $60,0.07$ & & $7,0.66$ & & $6,3 \cdot 22$ & & & & \\
\hline Model 1 & 1 & Ref. & 1.06 & $0.72,1.57$ & $1 \cdot 15$ & $0.78,1.68$ & 1.55 & $1.08,2.24$ & 0.018 & $1 \cdot 22$ & $1.07,1.40$ & 0.003 \\
\hline Model 2 & 1 & Ref. & 1.02 & $0.69,1.52$ & $1 \cdot 11$ & $0.75,1.64$ & 1.51 & $1.05,2 \cdot 18$ & 0.026 & 1.22 & $1.06,1.39$ & 0.005 \\
\hline Model 3 & 1 & Ref. & 1.22 & $0.80,1.88$ & 1.25 & $0.81,1.92$ & 1.83 & $1.21,2.79$ & 0.007 & 1.32 & $1.12,1.54$ & 0.001 \\
\hline Rice-wheat-fruits & & & & & & & & & & & & \\
\hline GDM & & & & & & & & & & & & \\
\hline$n$ & & 32 & & 69 & & 46 & & 51 & & & & \\
\hline$\%$ & & 1.9 & & $10 \cdot 0$ & & $6 \cdot 7$ & & $7 \cdot 4$ & & & & \\
\hline Mean & & 1.02 & & -0.01 & & 0.33 & & 0.69 & & & & \\
\hline Min, $\max$ & $-1 s$ & $1,-0.24$ & & $24,0.20$ & & $20,0.47$ & & $17,1.69$ & & & & \\
\hline Model 1 & 1 & Ref. & 0.83 & $0.59,1.16$ & 0.53 & $0.36,0.77$ & 0.59 & $0.41,0.85$ & $<0.001$ & 0.77 & $0.70,0.86$ & $<0.001$ \\
\hline Model 2 & 1 & Ref. & 0.82 & $0.58,1.15$ & 0.52 & $0.35,0.76$ & 0.60 & $0.41,0.86$ & 0.001 & 0.77 & $0.69,0.85$ & $<0.001$ \\
\hline Model 3 & 1 & Ref. & 0.87 & $0.60,1.26$ & 0.54 & $0.36,0.83$ & 0.72 & $0.48,1.08$ & 0.010 & 0.78 & $0.68,0.88$ & $<0.001$ \\
\hline
\end{tabular}

Q, quartile; Ref, reference.

* Mean (minimum, maximum) of scores for each dietary pattern. OR and $95 \% \mathrm{Cl}$ calculated by logistic regression.

† Model 1 was crude model. Model 2 was adjusted for other dietary patterns. Model 3 was adjusted for model $2+$ maternal age, ethnology, maternal education, average personal income, family history of diabetes, family history of obesity, smoking, alcohol, parity, pre-pregnancy BMI, weight gain before GDM diagnosis and total energy intake.

\section{Sensitivity analyses}

We randomly selected for different sizes of samples in TMCHC to conduct principal component analysis to identify factors, and the results remained similar, showing that dietary patterns during pregnancy derived in our study were reproducible and stable (data not shown).

\section{Discussion}

In this study, we identified five maternal dietary patterns during pregnancy and examined their associations with GDM in
Chinese women. Interestingly, there were several important findings about the relationship between dietary patterns and GDM. Firstly, higher adherence to the fish-meat-eggs pattern was associated with an increased risk of GDM and that the association was stronger in overweight women and in women with a family history of diabetes. In contrast, higher adherence to the rice-wheat-fruits pattern was associated with a significantly reduced risk of GDM. Each of these associations was independent of established risk factors for GDM. Secondly, consistent with the findings on the association between dietary patterns and GDM risk, we observed a positive association 
Table 4. Gestational diabetes mellitus (GDM) according to the quartiles $(\mathrm{Q})$ of intake of two dietary patterns and macronutrients intakes in Tongji Maternal and Child Health Cohort study ( $n$ 2755)†

(Odds ratios and $95 \%$ confidence inervals)

\begin{tabular}{|c|c|c|c|c|c|c|c|c|c|}
\hline & \multicolumn{2}{|c|}{ Q1 } & \multicolumn{2}{|r|}{ Q2 } & \multicolumn{2}{|r|}{ Q3 } & \multicolumn{2}{|r|}{ Q4 } & \multirow[b]{2}{*}{$P_{\text {trend }}$} \\
\hline & OR & $95 \% \mathrm{Cl}$ & OR & $95 \% \mathrm{Cl}$ & OR & $95 \% \mathrm{Cl}$ & OR & $95 \% \mathrm{Cl}$ & \\
\hline \multicolumn{10}{|l|}{ Fish-meat-eggs } \\
\hline Adjusted model & 1 & Ref. & 1.22 & $0.80,1.88$ & 1.25 & $0.81,1.92$ & 1.83 & $1 \cdot 21,2 \cdot 79$ & 0.007 \\
\hline Adjusted model + total fat (En\%) & 1 & Ref. & 1.17 & $0.76,1.80$ & $1 \cdot 15$ & $0.74,1.79$ & 1.62 & $1 \cdot 03,2.54$ & 0.049 \\
\hline Adjusted model + animal fat (En\%) & 1 & Ref. & $1 \cdot 19$ & $0.77,1.84$ & $1 \cdot 18$ & $0.75,1.87$ & 1.69 & $1.06,2.72$ & 0.037 \\
\hline Adjusted model + vegetable fat (En\%) & 1 & Ref. & 1.23 & $0.80,1.89$ & 1.28 & $0.83,1.97$ & 1.89 & $1 \cdot 24,2 \cdot 88$ & 0.004 \\
\hline Adjusted model + total protein intake (En\%) & 1 & Ref. & 1.13 & $0.73,1.74$ & 1.08 & $0.70,1.68$ & 1.42 & $0.90,2 \cdot 22$ & 0.162 \\
\hline Adjusted model + animal protein intake (En\%) & 1 & Ref. & $1 \cdot 15$ & $0.74,1.78$ & $1 \cdot 11$ & $0.71,1.75$ & 1.51 & $0.94,2.44$ & $0 \cdot 119$ \\
\hline Adjusted model + vegetable protein intake (En\%) & 1 & Ref. & 1.27 & $0.83,1.96$ & $1 \cdot 36$ & $0.88,2 \cdot 10$ & 2.06 & $1.34,3.17$ & 0.001 \\
\hline Adjusted model + total carbohydrate intake (En\%) & 1 & Ref. & 1.13 & $0.73,1.75$ & 1.09 & $0.69,1.71$ & 1.48 & $0.92,2.36$ & 0.129 \\
\hline Adjusted model + (total protein)/(total carbohydrate) (\%) & 1 & Ref. & 1.12 & $0.72,1.72$ & 1.06 & $0.68,1.65$ & 1.35 & $0 \cdot 84,2 \cdot 16$ & 0.263 \\
\hline \multicolumn{10}{|l|}{ Rice-wheat-fruits } \\
\hline Adjusted model & 1 & Ref. & 0.87 & $0.60,1.26$ & 0.54 & $0.36,0.83$ & 0.72 & $0.48,1.08$ & 0.010 \\
\hline Adjusted model + total fat (En\%) & 1 & Ref. & 0.88 & $0.61,1.29$ & 0.55 & $0.36,0.85$ & 0.73 & $0.48,1.09$ & 0.013 \\
\hline Adjusted model + animal fat (En\%) & 1 & Ref. & 0.87 & $0.60,1.26$ & 0.55 & $0.36,0.83$ & 0.72 & $0.48,1.08$ & 0.011 \\
\hline Adjusted model + vegetable fat (En\%) & 1 & Ref. & 0.89 & $0.61,1.30$ & 0.56 & $0.37,0.85$ & 0.73 & $0.49,1.10$ & 0.015 \\
\hline Adjusted model + total protein intake (En\%) & 1 & Ref. & 0.92 & $0.63,1.34$ & 0.60 & $0.39,0.92$ & 0.83 & $0.55,1.26$ & 0.105 \\
\hline Adjusted model + animal protein intake (En\%) & 1 & Ref. & 0.88 & $0.60,1.27$ & 0.56 & $0.36,0.85$ & 0.75 & $0.50,1.14$ & 0.032 \\
\hline Adjusted model + vegetable protein intake (En\%) & 1 & Ref. & 0.89 & $0.61,1.30$ & 0.57 & $0.37,0.87$ & 0.75 & $0.50,1.13$ & 0.037 \\
\hline Adjusted model + total carbohydrate intake (En\%) & 1 & Ref. & 0.90 & $0.62,1.31$ & 0.57 & $0.37,0.87$ & 0.75 & $0.50,1.13$ & 0.033 \\
\hline Adjusted model + (total protein)/(total carbohydrate) (\%) & 1 & Ref. & 0.94 & $0.64,1.37$ & 0.60 & $0.39,0.92$ & 0.82 & $0.54,1.24$ & 0.094 \\
\hline Total fat $(E n \%) \ddagger$ & 1 & Ref. & 1.47 & $0.96,2.27$ & 1.31 & $0.84,2.04$ & 1.85 & $1 \cdot 21,2 \cdot 81$ & 0.010 \\
\hline Animal fat $(E n \%) \ddagger$ & 1 & Ref. & 1.38 & $0.89,2.14$ & 1.76 & $1.15,2.69$ & 1.81 & $1 \cdot 18,2 \cdot 77$ & 0.003 \\
\hline Vegetable fat $(\mathrm{En} \%) \ddagger$ & 1 & Ref. & 1.00 & $0.64,1.57$ & 0.87 & $0.53,1.43$ & 1.36 & $0.81,2 \cdot 29$ & 0.244 \\
\hline Total protein intake (En\%)‡ & 1 & Ref. & $1 \cdot 70$ & $1.08,2.68$ & 1.60 & $1.01,2.53$ & 2.67 & $1 \cdot 71,4.14$ & $<0.001$ \\
\hline Animal protein intake (En\%)‡ & 1 & Ref. & 1.21 & $0.78,1.88$ & 1.55 & $1.01,2.36$ & 1.97 & $1.30,3.00$ & 0.001 \\
\hline Vegetable protein intake (En\%)‡ & 1 & Ref. & $1 \cdot 10$ & $0.74,1.65$ & 0.99 & $0.66,1.49$ & 1.07 & $0.71,1.60$ & 0.897 \\
\hline Total carbohydrate intake (En\%)‡ & 1 & Ref. & 0.73 & $0.50,1.05$ & 0.79 & $0.55,1.14$ & 0.43 & $0.28,0.66$ & 0.001 \\
\hline (Total protein)/(total carbohydrate) $(\%) \ddagger$ & 1 & Ref. & $1 \cdot 15$ & $0.71,1.86$ & 2.32 & $1.49,3.60$ & $2 \cdot 41$ & $1.56,3.72$ & $<0.001$ \\
\hline Per 1 percentage point increase $\neq$ & $1.05^{\star \star \star}$ & $1.03,1.07$ & & & & & & & \\
\hline Per 5 percentage point increasef & $1.26^{\star \star *}$ & $1.14,1.40$ & & & & & & & \\
\hline
\end{tabular}

Ref., reference; En\%, percentage energy.

${ }^{* \star \star} P<0.001$

† Adjusted model was adjusted for maternal age, ethnology, maternal education, average personal income, family history of diabetes, family history of obesity, smoking, alcohol, parity, pre-pregnancy BMI, weight gain before GDM diagnosis, other dietary patterns and total energy intake.

$\ddagger$ Adjusted for maternal age, ethnology, maternal education, average personal income, family history of diabetes, family history of obesity, smoking, alcohol, parity, pre-pregnancy BMI, weight gain before GDM diagnosis and total energy intake.

between the fish-meat-eggs score and plasma glucose levels and an inverse association between the rice-wheat-fruits score and plasma glucose levels. Moreover, we specifically designed to investigate the association of dietary patterns with GDM risk or plasma glucose levels with adjustment for dietary macronutrient intakes, and we found the association between dietary pattern and GDM may be at least partially attributed to intake of dietary protein and carbohydrate. The results suggested that a high-protein and low-carbohydrate dietary pattern in pregnancy was associated with a higher risk of GDM in Chinese women.

Several recent studies of Caucasian populations have reported that a maternal diet high in red meat, processed meat and eggs was associated with a higher risk of $\mathrm{GDM}^{(15,34)}$. Our results were in line with these studies, demonstrating that the fishmeat-eggs pattern - a maternal diet high in freshwater fish, red meat and eggs, similar to the Western pattern which was defined in the Nurses' Health Study II (NHSII) ${ }^{(15)}$ - was associated with a higher risk of GDM. Despite similarities in the association with dietary pattern and GDM, the contribution of red meat to fish-meat-eggs pattern in the present study was different from its contribution to Western pattern in NHSII, by the evidence that the strong association of fish-meat-eggs pattern with GDM risk remained significant in the present study after adjustment for red meat intake, but was no longer significant in the NHSII ${ }^{(15,23)}$. One possible reason for this may be that the red meat consumption (approximately $105 \mathrm{~g} / \mathrm{d}$ on average) in $\mathrm{NHSII}^{(23)}$ was substantially higher than the average daily intake $(49 \cdot 5 \mathrm{~g} / \mathrm{d})$ in our study.

In addition, the rice-wheat-fruits pattern was associated with a significantly reduced risk of GDM, which was also in line with the findings from the NHSII ${ }^{(15,16)}$. Women adherence to the prudent pattern characterised by a high intake of fruit, vegetables and green leafy vegetables were associated with decreased risks for $\mathrm{GDM}^{(15)}$. Importantly, our analysis of diet was conducted in the second trimester, which was different from the time in prepregnancy of NHSII, suggesting that our similar findings in pregnant Chinese women may confirm the association of dietary pattern with the risk of GDM found in NHSII. Moreover, a vegetable-fruit-rice-based dietary pattern resembled the rice-wheatfruits dietary pattern was observed to be associated with lower fasting blood glucose concentrations in a Multi-Ethnic Asian Cohort $^{(20)}$. 
Table 5. Associations between two dietary pattern scores and plasma glucose levels ( $n$ 2755)*

( $\beta$-Coefficients and $95 \%$ confidence intervals)

\begin{tabular}{|c|c|c|c|c|c|c|c|c|c|}
\hline \multirow[b]{2}{*}{ Dietary patterns } & \multicolumn{2}{|c|}{ Fasting plasma glucose } & \multirow[b]{2}{*}{$P$} & \multicolumn{2}{|c|}{ 1-h plasma glucose } & \multirow[b]{2}{*}{$P$} & \multicolumn{2}{|c|}{ 2-h plasma glucose } & \multirow[b]{2}{*}{$P$} \\
\hline & $\beta$ & $95 \% \mathrm{Cl}$ & & $\beta$ & $95 \% \mathrm{Cl}$ & & $\beta$ & $95 \% \mathrm{Cl}$ & \\
\hline \multicolumn{10}{|l|}{ Fish-meat-eggs } \\
\hline Crude & 0.013 & $-0.001,0.027$ & 0.072 & 0.102 & $0.046,0.158$ & $<0.001$ & 0.051 & $0.008,0.094$ & 0.020 \\
\hline Adjusted model & 0.018 & $0.004,0.033$ & 0.012 & 0.136 & $0.077,0.195$ & $<0.001$ & 0.074 & $0.028,0.120$ & 0.001 \\
\hline Adjusted model + total fat (En\%) & 0.014 & $-0.002,0.029$ & 0.086 & 0.140 & $0.075,0.204$ & $<0.001$ & 0.076 & $0.026,0.126$ & 0.003 \\
\hline Adjusted model + animal fat (En\%) & 0.011 & $-0.006,0.027$ & 0.210 & 0.132 & $0.063,0.200$ & $<0.001$ & 0.058 & $0.006,0.111$ & 0.030 \\
\hline Adjusted model + vegetable fat (En\%) & 0.018 & $0.003,0.032$ & 0.015 & 0.134 & $0.074,0.193$ & $<0.001$ & 0.071 & $0.025,0.117$ & 0.003 \\
\hline Adjusted model + total protein intake (En\%) & 0.008 & $-0.008,0.024$ & 0.310 & 0.116 & $0.051,0.180$ & $<0.001$ & 0.055 & $0.005,0.105$ & 0.031 \\
\hline Adjusted model + animal protein intake (En\%) & 0.005 & $-0.012,0.022$ & 0.553 & 0.124 & $0.052,0.195$ & 0.001 & 0.055 & $0.000,0.110$ & 0.048 \\
\hline Adjusted model + vegetable protein intake (En\%) & 0.020 & $0.005,0.035$ & 0.009 & 0.147 & $0.085,0.209$ & $<0.001$ & 0.080 & $0.032,0.128$ & 0.001 \\
\hline Adjusted model + total carbohydrate intake (En\%) & 0.010 & $-0.006,0.027$ & 0.209 & 0.134 & $0.066,0.201$ & $<0.001$ & 0.070 & $0.018,0.122$ & 0.008 \\
\hline Adjusted model + (total protein)/(total carbohydrate) $(\%)$ & 0.006 & $-0.010,0.023$ & 0.472 & 0.117 & $0.049,0.185$ & 0.001 & 0.056 & $0.004,0.109$ & 0.036 \\
\hline \multicolumn{10}{|l|}{ Rice-wheat-fruits } \\
\hline Crude & -0.021 & $-0.035,-0.007$ & 0.003 & -0.135 & $-0.191,-0.080$ & $<0.001$ & -0.114 & $-0.156,-0.071$ & $<0.001$ \\
\hline Adjusted model & -0.014 & $-0.028,-0.001$ & 0.049 & -0.100 & $-0.158,-0.042$ & 0.001 & -0.093 & $-0.137,-0.048$ & $<0.001$ \\
\hline Adjusted model + total fat (En\%) & -0.013 & $-0.027,0.001$ & 0.064 & -0.099 & $-0.158,-0.041$ & 0.001 & -0.092 & $-0.137,-0.048$ & $<0.001$ \\
\hline Adjusted model + animal fat (En\%) & -0.014 & $-0.028,0.000$ & 0.058 & -0.099 & $-0.157,-0.040$ & 0.001 & -0.091 & $-0.136,-0.046$ & $<0.001$ \\
\hline Adjusted model + vegetable fat (En\%) & -0.014 & $-0.028,-0.001$ & 0.047 & -0.102 & $-0.160,-0.044$ & 0.001 & -0.096 & $-0.140,-0.051$ & $<0.001$ \\
\hline Adjusted model + total protein intake (En\%) & -0.010 & $-0.024,0.004$ & 0.168 & -0.087 & $-0.146,-0.028$ & 0.004 & -0.082 & $-0.128,-0.037$ & $<0.001$ \\
\hline Adjusted model + animal protein intake (En\%) & -0.012 & $-0.026,0.002$ & 0.100 & -0.094 & $-0.152,-0.035$ & 0.002 & -0.087 & $-0.132,-0.042$ & $<0.001$ \\
\hline Adjusted model + vegetable protein intake (En\%) & -0.014 & $-0.028,0.000$ & 0.058 & -0.098 & $-0.156,-0.039$ & 0.001 & -0.092 & $-0.136,-0.047$ & $<0.001$ \\
\hline Adjusted model + total carbohydrate intake (En\%) & -0.012 & $-0.027,0.002$ & 0.085 & -0.097 & $-0.155,-0.038$ & 0.001 & -0.090 & $-0.135,-0.045$ & $<0.001$ \\
\hline Adjusted model + total protein)/(total carbohydrate) (\%) & -0.010 & $-0.024,0.005$ & 0.194 & -0.086 & $-0.146,-0.027$ & 0.005 & -0.082 & $-0.128,-0.036$ & $<0.001$ \\
\hline Total fat intake $(E n \%) \dagger$ & 0.003 & $0.001,0.005$ & 0.008 & 0.009 & $0.001,0.018$ & 0.034 & 0.005 & $-0.001,0.012$ & 0.105 \\
\hline Animal fat (En\%)† & 0.003 & $0.001,0.006$ & 0.005 & 0.014 & $0.004,0.023$ & 0.005 & 0.011 & $0.004,0.019$ & 0.003 \\
\hline Vegetable fat (En\%)† & 0.000 & $-0.004,0.004$ & 0.986 & -0.005 & $-0.023,0.013$ & 0.565 & -0.009 & $-0.023,0.004$ & 0.184 \\
\hline Total protein intake (En\%)† & 0.014 & $0.007,0.021$ & $<0.001$ & 0.060 & $0.030,0.089$ & $<0.001$ & 0.049 & $0.026,0.071$ & $<0.001$ \\
\hline Animal protein intake $(\mathrm{En} \%) \dagger$ & 0.012 & $0.006,0.019$ & $<0.001$ & 0.054 & $0.026,0.081$ & $<0.001$ & 0.042 & $0.020,0.063$ & $<0.001$ \\
\hline Vegetable protein intake (En\%)† & 0.000 & $-0.010,0.010$ & 0.967 & 0.001 & $-0.041,0.043$ & 0.966 & 0.005 & $-0.027,0.038$ & 0.747 \\
\hline Total carbohydrate intake (En\%)† & -0.003 & $-0.005,-0.001$ & 0.001 & -0.012 & $-0.019,-0.004$ & 0.003 & -0.008 & $-0.014,-0.002$ & 0.011 \\
\hline (Total protein)//total carbohydrate) $(\%) \dagger$ & 0.005 & $0.002,0.007$ & $<0.001$ & 0.020 & $0.011,0.030$ & $<0.001$ & 0.015 & $0.008,0.022$ & $<0.001$ \\
\hline
\end{tabular}

En\%, percentage energy; GDM, gestational diabetes mellitus.

* Adjusted model was adjusted for maternal age, ethnology, maternal education, average personal income, family history of diabetes, family history of obesity, smoking, alcohol, parity, pre-pregnancy BMI, weight gain before GDM diagnosis, other dietary patterns and total energy intake. Values are linear regression coefficients and $95 \% \mathrm{Cl}$.

† Adjusted for maternal age, ethnology, maternal education, average personal income, family history of diabetes, family history of obesity, smoking, alcohol, parity, pre-pregnancy BMI, weight gain before GDM diagnosis and total energy intake. Values are linear regression coefficients and $95 \% \mathrm{Cl}$. 
Previous studies have indicated that macronutrient intake was associated with $\operatorname{GDM}^{(10,11,13,14,22)}$. Our results were in line with previous findings that a higher pre-pregnancy intake of protein or fat, in particular animal origin, was significantly associated with a greater risk of $\mathrm{GDM}^{(13,14)}$, and a lower intake of carbohydrate during mid-pregnancy was associated with an increased risk of GDM later in pregnancy ${ }^{(11)}$. In addition, a major characteristic of the fish-meat-eggs pattern is high intakes of total protein, animal protein, total fat and animal fat, while a major characteristic of rice-wheat-fruits pattern is a high intake of carbohydrate. In the present study, the positive association between fish-meat-eggs pattern and GDM risk became non-significant after adjustment for total protein, animal protein and total carbohydrate intake, respectively, and the inverse association between rice-wheat-fruits pattern and GDM became non-significant after adjustment for total protein, and ratio of total protein to total carbohydrate, respectively, suggesting that the observed association of dietary patterns with GDM may have been due to the distribution intakes of protein and carbohydrate across quartiles of the dietary patterns. Similar to our results, in the NHSII, a pre-pregnancy low-carbohydrate dietary pattern with high protein and fat from animal-food sources was positively associated with GDM risk ${ }^{(23)}$. However, the associations between dietary patterns and GDM were not significantly modified by adjusting for intake of total fat or animal fat, although there was a positive association of total fat intake or animal fat intake with GDM risk in the present study.

Our findings are biologically plausible, although the underlying mechanisms remain to be elucidated. First, dietary proteins and amino acids are important modulators of glucose metabolism and insulin sensitivity ${ }^{(14,35)}$, and a short-term increase in plasma amino acid concentrations has been shown to directly induce insulin resistance in skeletal muscle and stimulate endogenous glucose production ${ }^{(36)}$. Further research is needed to evaluate the associations between dietary patterns and insulin resistance such as insulin values and homeostasis model assessment of insulin resistance (HOMAIR). Second, prospective studies provided evidence that branched-chain amino acids (BCAA), aromatic amino acids, were associated with the incidence of prediabetes and type 2 diabetes $^{(37)}$. Moreover, metabolomics studies have demonstrated that increased fasting concentrations of circulating BCAA are associated with an increased risk of type 2 diabetes and insulin resistance in humans and in some rodent models ${ }^{(38)}$. Finally, another potential explanation is related to the complex carbohydrate. In the present study, average $61.8 \%$ of total carbohydrates are from grains, suggesting the components of carbohydrate were mainly complex carbohydrates. A prospective, randomised crossover trial demonstrated that a shortterm diet with liberalised complex carbohydrate and limited fat effectively controlled maternal glycemia and postprandial lipids ${ }^{(39)}$. Moreover, a high-carbohydrate diet represents combinations of a higher content of carbohydrate and lower contents of fat and protein from the diet, which may also explain the associations found between the dietary patterns and GDM risk in the present study ${ }^{(13,14)}$.

In addition, we also examined the other nutrients' potential contributions on the association between dietary patterns and risk of GDM, including total dietary fibre, dietary vitamin C and dietary vitamin $\mathrm{E}$ which are shown to reduce GDM risk in previous studies $^{(11,40,41)}$, and cholesterol and haem Fe which might contribute to the increased risk of $\mathrm{GDM}^{(9)}$. However, the association between dietary patterns and GDM risk remained strong after adjusting for dietary fibre, dietary vitamin C, dietary vitamin E and haem Fe, suggesting that these nutrients were not contributors to the association of dietary pattern with GDM risk in the present study. Interestingly, the strong association of fish-meat-eggs pattern with GDM risk became non-significant after further adjustment for dietary cholesterol intake, which mainly came from red meat and eggs, indicating that cholesterol might also be related to the pathogenesis of GDM. Further studies are warranted to validate this interaction and explore potential mechanisms.

Considering the role of a high pre-pregnancy BMI and the family history of diabetes in the development of $\mathrm{GDM}^{(42,43)}$, the stratified analysis were performed in the present study. The results showed that the association between the fish-meat-eggs pattern and the risk of GDM was stronger in overweight women and in women with a family history of diabetes, which is clinically important. Understanding dietary patterns in relation to GDM in obese pregnant women will provide potential targets for future interventions to improve pregnancy outcomes ${ }^{(42)}$. However, the protective effect of the rice-wheat-fruits pattern was not significant in the high BMI group or in women with a family history of diabetes, suggesting that for overweight women or women with a family history of diabetes, other environmental factors or genetic components may play a more important role than rice-wheat-fruits pattern in the development of $\mathrm{GDM}^{(43,44)}$.

The strengths of our study include its prospective design, the uniform criteria for diagnosis of GDM and the availability of abundant information on covariates that allowed us to diminish potential confounders as much as possible ${ }^{(16)}$. Another strength is the prospective assessment of the pregnancy diet, which means that women completed an FFQ before their OGTT; thus, a diagnosis of GDM could not affect dietary reporting. In addition, the FFQ used in our study has been validated in a TMCHC subsample ${ }^{(24)}$. Finally, a key strength is the focus on dietary patterns, rather than individual dietary components, and the design of investigating the association between dietary pattern and GDM with adjustment for dietary macronutrient intakes.

We also acknowledge that our study has limitations. First, the data-driven approach of deriving dietary patterns is observational, making a comparison with results from other studies challenging. However, the results of sensitivity analysis by using different sample size from the TMCHC demonstrated the internal validity and reproducibility (data not shown). In addition, the rice-wheat-fruits and fish-meat-eggs patterns shared some similarities with the prudent diets and Western diets, respectively, and our findings are largely consistent with most existing studies on prudent diets and Western diets ${ }^{(14,16,33,34)}$. Second, our study population consisted mostly of Han Chinese, thus we are unable to ascertain whether the association is similar in other ethnic groups. However, the relative homogeneity of our population advantageously reduced unmeasured confounding. Third, self-reported weight before pregnancy may lead to under-estimation of overweight or obese ${ }^{(45)}$. However, the self- 
reported pre-pregnancy weight is widely used in observational studies $^{(29,46)}$. Fourth, a relative shortened FFQ consisting of sixty-one food items was used to classify dietary intake in the present study. Finally, despite careful consideration of the known risk factors and potential confounding factors, residual confounding cannot be ruled out.

In conclusion, the present prospective study, which was conducted in central China, showed that a maternal dietary pattern characterised by high protein and low carbohydrate intake in pregnancy was associated with a higher risk of GDM. These findings of our study provide important clues for dietary guidance during pregnancy to prevent GDM.

\section{Acknowledgements}

The authors gratefully acknowledge the cooperation of the pregnant women who took part in this study. The authors also thank the staff of Hubei Maternal and Child Health Hospital, Jiang'an Maternal and Child Health Hospital and The Central Hospital of Wuhan for their considerable assistance with many aspects of this study. The authors are sincerely grateful to all members of the TMCHC Study Group.

This work was supported by the National Program on Basic Research Project of China (no. 2013FY114200) for N. Yang, and the Dietary Nutrition Research and Education Fundation of Danone Nutrition Center in China (DIC2015-08) for X. Y.

The authors' responsibilities are as follows: X. Z., X. Y. and N. Yang designed the research. L. H., X. Y. and N. Yang supervised the study conduct. X. Z., R. C., C. Z., J. W., X. L., Q. L., W. C., N. Yi, M. X., H. Y., G. X. and W. H. conducted the research. X. Z. and X. Y. analysed and interpreted the data. X. Z. wrote the manuscript. X. Y. and N. Yang reviewed and edited the manuscript and contributed to discussion. N. Yang had primary responsibility for final content. All authors read and approved the final version of the manuscript.

None of the authors has any conflicts of interest to declare.

\section{Supplementary materials}

For supplementary material/s referred to in this article, please visit https://doi.org/10.1017/S0007114518002453

\section{References}

1. Reece EA, Leguizamón G \& Wiznitzer A (2009) Gestational diabetes: the need for a common ground. Lancet $\mathbf{3 7 3}$, 1789-1797.

2. Bentley-Lewis R (2009) Gestational diabetes mellitus as a herald of type 2 diabetes mellitus: an opportunity for a lifetime. Lancet 373, 1738-1740.

3. Kessous R, Shoham-Vardi I, Pariente G, et al. (2013) An association between gestational diabetes mellitus and long-term maternal cardiovascular morbidity. Heart 99, 1118-1121.

4. Ben-Haroush A, Yogev Y \& Hod M (2004) Epidemiology of gestational diabetes mellitus and its association with type 2 diabetes. Diabet Med 21, 103-113.

5. Buckley BS, Harreiter J, Damm P, et al. (2012) Gestational diabetes mellitus in Europe: prevalence, current screening practice and barriers to screening. A review. Diabet Med 29 , 844-854.

6. Dabelea D, Snell-Bergeon JK, Hartsfield CL, et al. (2005) Increasing prevalence of gestational diabetes mellitus (GDM) over time and by birth cohort: Kaiser Permanente of Colorado GDM Screening Program. Diabetes Care 28, 579-584.

7. Leng J, Shao P, Zhang C, et al. (2015) Prevalence of gestational diabetes mellitus and its risk factors in Chinese pregnant women: a prospective population-based study in Tianjin, China. PLOS ONE 10, e0121029.

8. Zhang C \& Ning Y (2011) Effect of dietary and lifestyle factors on the risk of gestational diabetes: review of epidemiologic evidence. Am J Clin Nutr 94, Suppl, 1975S-1979S.

9. Schoenaker DA, Mishra GD, Callaway LK, et al. (2016) The role of energy, nutrients, foods, and dietary patterns in the development of gestational diabetes mellitus: a systematic review of observational studies. Diabetes Care 39, 16-23.

10. Radesky JS, Oken E, Rifas-Shiman SL, et al. (2008) Diet during early pregnancy and development of gestational diabetes. Paediatr Perinat Epidemiol 22, 47-59.

11. Ley SH, Hanley AJ, Retnakaran R, et al. (2011) Effect of macronutrient intake during the second trimester on glucose metabolism later in pregnancy. Am J Clin Nutr $\mathbf{9 4}$, $1232-1240$.

12. Bao W, Tobias DK, Olsen SF, et al. (2014) Pre-pregnancy fried food consumption and the risk of gestational diabetes mellitus: a prospective cohort study. Diabetologia 57, 2485-2491.

13. Bowers K, Tobias DK, Yeung E, et al. (2012) A prospective study of prepregnancy dietary fat intake and risk of gestational diabetes. Am J Clin Nutr 95, 446-453.

14. Bao W, Bowers K, Tobias DK, et al. (2013) Pre-pregnancy dietary protein intake, major dietary protein sources, and the risk of gestational diabetes mellitus: a prospective cohort study. Diabetes Care 36, 2001-2008.

15. Zhang C, Schulze MB, Solomon CG, et al. (2006) A prospective study of dietary patterns, meat intake and the risk of gestational diabetes mellitus. Diabetologia 49, 2604-2613.

16. Tobias DK, Zhang C, Chavarro J, et al. (2012) Pre-pregnancy adherence to dietary patterns and lower risk of gestational diabetes mellitus. Am J Clin Nutr 96, 289-295.

17. Shin D, Lee KW \& Song WO (2015) Dietary patterns during pregnancy are associated with risk of gestational diabetes mellitus. Nutrients 7, 9369-9382.

18. Tryggvadottir EA, Medek H, Birgisdottir BE, et al. (2016) Association between healthy maternal dietary pattern and risk for gestational diabetes mellitus. Eur J Clin Nutr 70, 237-242.

19. Karamanos B, Thanopoulou A, Anastasiou E, et al. (2014) Relation of the Mediterranean diet with the incidence of gestational diabetes. Eur J Clin Nutr 68, 8-13.

20. de Seymour J, Chia A, Colega M, et al. (2016) Maternal dietary patterns and gestational diabetes mellitus in a multi-ethnic Asian cohort: the GUSTO study. Nutrients 8, 574 .

21. He JR, Yuan MY, Chen NN, et al. (2015) Maternal dietary patterns and gestational diabetes mellitus: a large prospective cohort study in China. Br J Nutr 113, 1292-1300.

22. Pang WW, Colega M, Cai S, et al. (2017) Higher maternal dietary protein intake is associated with a higher risk of gestational diabetes mellitus in a multiethnic Asian cohort. J Nutr 147, 653-660.

23. Bao W, Bowers K, Tobias DK, et al. (2014) Prepregnancy lowcarbohydrate dietary pattern and risk of gestational diabetes mellitus: a prospective cohort study. Am J Clin Nutr 99, 1378-1384

24. Zhang H, Qiu X, Zhong C, et al. (2015) Reproducibility and relative validity of a semi-quantitative food frequency questionnaire for Chinese pregnant women. Nutr J 14, 56. 
25. Englund-Ögge L, Brantsæter AL, Sengpiel V, et al. (2014) Maternal dietary patterns and preterm delivery: results from large prospective cohort study. BMJ 348, g1446.

26. National Institute of Nutrition and Food Safety, China Centers for Disease Control (2009) China Food Composition, 2nd ed. Beijing: Peking University Medical Press.

27. Willett WC, Howe GR \& Kushi LH (1997) Adjustment for total energy in take in epidemiologic studies. Am J Clin Nutr $\mathbf{6 5}$, Suppl, 1220S-1228S.

28. International Association of Diabetes and Pregnancy Study Groups Consensus Panel, Metzger BE, Gabbe SG, et al. (2010) International association of diabetes and pregnancy study groups recommendations on the diagnosis and classification of hyperglycemia in pregnancy. Diabetes Care 33, 676-682.

29. Rimm EB, Stampfer MJ, Colditz GA, et al. (1990) Validity of self-reported waist and hip circumferences in men and women. Epidemiology 1, 466-473.

30. Chen CM (2008) Overview of obesity in mainland China. Obes Rev 1, 14S-21S

31. Hu FB (2002) Dietary pattern analysis: a new direction in nutritional epidemiology. Curr Opin Lipidol 13, 3-9.

32. Moeller SM, Reedy J, Millen AE, et al. (2007) Dietary patterns: challenges and opportunities in dietary patterns research an Experimental Biology workshop, April 1, 2006. J Am Diet Assoc 107, 1233-1239.

33. Chia AR, de Seymour JV, Colega M, et al. (2016) A vegetable, fruit, and white rice dietary pattern during pregnancy is associated with a lower risk of preterm birth and larger birth size in a multiethnic Asian cohort: the Growing Up in Singapore Towards Healthy Outcomes (GUSTO) cohort study. Am J Clin Nutr 104, 1416-1423.

34. Qiu C, Frederick IO, Zhang C, et al. (2011) Risk of gestational diabetes mellitus in relation to maternal egg and cholesterol intake. Am J Epidemiol 173, 649-658.

35. Tremblay F, Lavigne C, Jacques H, et al. (2007) Role of dietary proteins and amino acids in the pathogenesis of insulin resistance. Annu Rev Nutr 27, 293-310.
36. Promintzer M \& Krebs M (2006) Effects of dietary protein on glucose homeostasis. Curr Opin Clin Nutr Metab Care $\mathbf{9}$, 463-468.

37. Guasch-Ferré M, Hruby A, Toledo E, et al. (2016) Metabolomics in prediabetes and diabetes: a systematic review and meta-analysis. Diabetes Care 39, 833-846.

38. Lynch CJ \& Adams SH (2014) Branched-chain amino acids in metabolic signalling and insulin resistance. Nat Rev Endocrinol 10, 723-736.

39. Hernandez TL, Van Pelt RE, Anderson MA, et al. (2014) A higher-complex carbohydrate diet in gestational diabetes mellitus achieves glucose targets and lowers postprandial lipids: a randomized crossover study. Diabetes Care 37, 1254-1262.

40. Zhang C, Williams MA, Sorensen TK, et al. (2004) Maternal plasma ascorbic acid (vitamin C) and risk of gestational diabetes mellitus. Epidemiology 15, 597-604.

41. Parast VM \& Paknahad Z (2017) Antioxidant status and risk of gestational diabetes mellitus: a case-control study. Clin Nutr Res 6, 81-88.

42. Torloni MR, Betrán AP, Horta BL, et al. (2009) Prepregnancy BMI and the risk of gestational diabetes: a systematic review of the literature with meta-analysis. Obes Rev 10, 194-203.

43. Yang H, Wei Y, Gao X, et al. (2009) Risk factors for gestational diabetes mellitus in Chinese women: a prospective study of 16,286 pregnant women in China. Diabet Med 26, 1099-1104.

44. Zhang C, Bao W, Rong Y, et al. (2013) Genetic variants and the risk of gestational diabetes mellitus: a systematic review. Hum Reprod Update 19, 376-390.

45. Bolton-Smith C, Woodward M, Tunstall-Pedoe H, et al. (2000) Accuracy of the estimated prevalence of obesity from self reported height and weight in an adult Scottish population. J Epidemiol Community Health 54, 143-148.

46. Lederman SA \& Paxton A (1998) Maternal reporting of prepregnancy weight and birth outcome: consistency and completeness compared with the clinical record. Matern Child Health J 2, 123-126. 